\title{
PREVALENCIA DE Giardia sp. EN Canis familiaris DE LOS DISTRITOS DE LA PROVINCIA CONSTITUCIONAL DEL CALLAO
}

\author{
William Araujo T. ${ }^{1}$, Amanda Chávez V. ${ }^{2,3}$, Eva Casas A. ${ }^{2}$ y Néstor Falcón P. ${ }^{4}$
}

\section{Abstract}

The aim of this study was to determine the Giardia sp. prevalence in household dog populations on the six districts of the Provincia Constitucional del Callao. Fecal samples were collected from 385 apparently healthy dogs of various ages and processed by the spontaneous sedimentation technique. The results indicated the $9.4 \pm 2 \%(36 / 385)$ of the canine population were positive to Giardia sp. cysts. Relationships between cysts detection and fecal samples characteristics, and origin, sex and age of dogs were analyzed by logistic regression. A significant statistical relationship was found between Giardia sp. detection and physical characteristics of fecal samples. Results show a moderate Giardia sp. infection in dogs which evidences a zoonotic risk that highlight the necessity of setting up educational programmes in order to prevent Giardia sp. Transmission, especially to infant population.

Key words: Callao, zoonosis, dogs, diarrhoea, giardiasis

\section{RESUMEN}

El objetivo de este trabajo fue determinar la prevalencia de Giardia sp. en la población canina doméstica de los 6 distritos que conforman la Provincia Constitucional del Callao. Se colectaron 385 muestras fecales de perros, aparentemente normales, de ambos sexos, de diferentes edades y de acuerdo a la zona en donde habitaban sus propietarios. Las muestras fueron procesadas mediante la técnica de sedimentación espontánea encontrándose una prevalencia de $9.4 \pm 2 \%$ de Giardia sp. Se cuantificó la asociación entre la presencia del parásito y las características físicas de las heces, estrato distrital de procedencia, sexo y edad del canino mediante la prueba de regresión logística, determinándose una relación estadísticamente significativa entre el hallazgo de quistes de Giardia sp. y las características físicas de las muestras. Los resultados denotan una parasitosis moderada de Giardia sp. en los caninos, evidenciando un riesgo zoonótico, por lo que se hace necesario el establecimiento de programas educativos para prevenir la posibilidad de contagio, especialmente en la población infantil.

Palabras clave: Callao, zoonosis, perros, diarrea, giardiasis

\author{
${ }^{1}$ Práctica privada \\ ${ }^{2}$ Laboratorio de Microbiología y Parasitología, FMV-UNMSM \\ ${ }^{3}$ E-mail: a_chavez_g@hotmail.com \\ ${ }^{4}$ Laboratorio de Medicina Veterinaria Preventiva, FMV-UNMSM
}




\section{INTRODUCCIÓN}

Giardia es un protozoario flagelado de ciclo directo que puede infectar al hombre y a la mayoría de los animales. Es el parásito intestinal más frecuentemente diagnosticado en los laboratorios de Salud Pública de los E.U.A. (Furness et al., 2000) y es altamente prevalente en muchas regiones del mundo (Stevens, 1982); especialmente donde hay una pobre condición de vida y bajos niveles de higiene (Meloni et al., 1993).

En el Perú se han realizado estudios sobre la presencia del parásito en adultos y niños en una comunidad rural, encontrándose una prevalencia de 3.3\% (Maco et al., 2002). En hospitales peruanos se ha encontrado un $32 \%$ en pacientes inmunocomprometidos (Uribe et al., 1997); además se ha determinado que la Giardia se encuentra entre los agentes enteropatogénicos causantes de diarrea aguda y persistente en niños (López et al., 1996).

La Giardia puede transmitirse al ser humano por los animales de compañía. Sin embargo, son escasos los estudios que existen en el país sobre la prevalencia de este protozoo en mascotas caninas (Zárate, 2003). Se conoce que la Provincia Constitucional del Callao alberga cerca de cien mil canes por lo que el objetivo del estudio fue determinar la prevalencia de Giardia sp. en los caninos de los distritos de la Provincia Constitucional del Callao.

\section{Materiales y Métodos}

El tamaño mínimo muestral se determinó mediante la fórmula para estimar una proporción basada en una distribución normal, con $95 \%$ de confianza y $5 \%$ de precisión, y con una prevalencia referencial de 15.69\% (Zárate et al., 2003).

Entre octubre del 2001 y julio del 2002 se obtuvieron 385 muestras fecales de pe- rros aparentemente sanos, escogidos aleatoriamente y provenientes de los 6 distritos que conforman la Provincia Constitucional del Callao. Los animales fueron agrupados en cuatro grupos etarios para fines de análisis: cachorros hasta los 6 meses de edad, cachorros de 6 a $<12$ meses, adultos de 1 a $<10$ años y gerontes $>10$ años. Para considerar el estrato distrital de procedencia se utilizó el Índice de Necesidades Básicas Insatisfechas usado por la DISA-I para la elaboración de planes y servicios de la población (DISA ICallao, 2001a).

Las muestras fecales fueron analizadas mediante la técnica de sedimentación espontánea (Tello, 1998) para confirmar la presencia o ausencia de quistes o trofozoitos de Giardia sp.

Se cuantificó la asociación entre la ocurrencia de quistes de Giardia sp. en las muestras fecales con la edad y sexo de los animales, características físicas de las heces y distritos de procedencia de las muestras mediante, utilizando la prueba de regresión logística.

\section{Resultados}

El Cuadro 1 muestra la prevalencia de Giardia sp. en perros de los distritos de la Provincia Constitucional del Callao. Se encontró que 36 de los 385 animales evaluados fueron positivos denotando una prevalencia de $9.4 \pm 2.9 \%$. En los distritos de La Punta y La Perla se encontraron las frecuencias más altas $(16.7 \%)$ y más bajas $(3.1 \%)$, respectivamente.

Los resultados indican un elevado nivel de positividad (42.0\%) en muestras de heces diarreicas o pastosas, en tanto que sólo $2.2 \%$ en muestras de heces aparentemente normales. La prueba de regresión logística (Cuadro 2) mostró una relación estadística significativa entre la detección de Giardia sp. y las características físicas de las heces. 
Cuadro 1. Prevalencia de Giardia sp. en Canis familiares de acuerdo a las variables sexo y edad del perro, estrato social de procedencia y características físicas de las heces en los distritos de la Provincia Constitucional del Callao, mediante la técnica de sedimentación espontánea (2001-2002)

\begin{tabular}{|c|c|c|c|c|c|}
\hline & \multicolumn{2}{|c|}{ Caninos } & \multirow{2}{*}{\multicolumn{3}{|c|}{$\% \pm \mathrm{IC}^{1}$}} \\
\hline & Total & Positivos & & & \\
\hline \multicolumn{6}{|l|}{ Distrito } \\
\hline La Punta & 06 & 01 & 16.7 & \pm & 29.8 \\
\hline La Perla & 32 & 01 & 3.1 & \pm & 6.0 \\
\hline Bellavista & 36 & 03 & 8.3 & \pm & 9.0 \\
\hline Callao & 195 & 22 & 11.3 & \pm & 4.4 \\
\hline C. de la Legua & 18 & 02 & 11.1 & \pm & 14.5 \\
\hline Reynoso & & & & & \\
\hline Ventanilla & 98 & 07 & 7.1 & \pm & 5.1 \\
\hline \multicolumn{6}{|l|}{ Sexo } \\
\hline Hembra & 207 & 21 & 10.1 & \pm & 4.1 \\
\hline Macho & 178 & 15 & 8.4 & \pm & 4.1 \\
\hline \multicolumn{6}{|l|}{ Edad } \\
\hline$<6$ meses & 111 & 12 & 10.8 & \pm & 5.8 \\
\hline $6 a<12 m$ & 95 & 8 & 8.4 & \pm & 5.6 \\
\hline $1 \mathrm{a}<10$ años & 137 & 12 & 8.8 & \pm & 4.7 \\
\hline$>10$ años & 42 & 4 & 9.5 & \pm & 4.5 \\
\hline \multicolumn{6}{|l|}{ Estratos distritales } \\
\hline I & 74 & 5 & 6.8 & \pm & 5.7 \\
\hline II & 213 & 24 & 11.3 & \pm & 4.2 \\
\hline III & 98 & 7 & 7.1 & \pm & 5.1 \\
\hline \multicolumn{6}{|l|}{ Muestra fecal } \\
\hline Normal & 316 & 7 & 2.2 & \pm & 1.6 \\
\hline Diarreica & 69 & 29 & 42.0 & \pm & 11.7 \\
\hline Total & 385 & 36 & 9.4 & \pm & 2.9 \\
\hline
\end{tabular}

${ }^{1}$ Tasa de prevalencia \pm intervalo de confianza del 95\%

El Cuadro 1 muestra además las prevalencias de Giardia sp. en caninos según el estrato distrital de procedencia con resultados bastante similares entre ellos. La prueba de regresión logística (Cuadro 2) indicó únicamente una tendencia estadística entre la presencia de Giardia sp. y el estrato distrital de procedencia de la muestra. Por otro lado, no se encontraron diferencias importantes en las prevalencias de acuerdo al sexo o edad.

\section{Discusión}

La giardiosis es una enfermedad producida por el protozoo Giardia sp., agente causal de diversos cuadros gastrointestinales en animales y en el hombre. Los individuos infectados pueden permanecer asintomáticos o presentar severos cuadros diarreicos.

Debido al estrecho contacto de los perros con sus dueños, surge la posibilidad de 
Cuadro 2. Prueba de regresión logística de los resultados obtenidos para la determinación de Giardia sp. mediante la técnica de sedimentación espontánea en muestras fecales de caninos de la Provincia Constitucional del Callao (2001-2002)

\begin{tabular}{|c|c|c|c|c|}
\hline Variable & & $\begin{array}{c}\text { Nivel de } \\
\text { Significancia }\end{array}$ & $\begin{array}{l}\text { Odss Ratio } \\
\text { (OR) }\end{array}$ & $\mathrm{IC}^{1}$ \\
\hline \multicolumn{5}{|l|}{ Sexo } \\
\hline & Hembra & - & 1 & - \\
\hline & Macho & 0.282 & 1.6 & $(0.68-3.76)$ \\
\hline \multicolumn{5}{|l|}{ Edad } \\
\hline & Cachorro (<6 meses) & - & 1 & - \\
\hline & Cachorro (6-12 meses) & 0.943 & 1 & $(0.31-3.48)$ \\
\hline & Adulto (>1 <10 años) & 0.912 & 0.95 & $(0.38-2.38)$ \\
\hline & Viejo (>10años) & 0.794 & 1.20 & $(0.31-4.68)$ \\
\hline \multicolumn{5}{|c|}{ Estrato distrital } \\
\hline & III & - & 1 & - \\
\hline & I & 0.308 & 2.18 & $(0.49-9.79)$ \\
\hline & II & 0.024 & 3.29 & $(1.172-9.216)$ \\
\hline \multicolumn{5}{|c|}{ Muestra fecal } \\
\hline & Normal & - & 1 & - \\
\hline & Diarreica & 0.000 & 38.6 & $(15.03-96.36)$ \\
\hline
\end{tabular}

${ }^{1}$ Intervalo de confianza del 95\%

una infección cruzada por lo que resulta importante determinar la presencia de Giardia sp. en caninos, ya que constituye un riesgo para la salud de la población. En tal sentido, se determinó la prevalencia de este protozoo en los caninos de la Provincia Constitucional del Callao, que alberga cerca de 100,000 perros en sus 6 distritos. Para tal fin, se evaluaron 385 muestras de heces mediante la técnica de sedimentación espontánea, obteniendo una prevalencia de $9.5 \pm 2.9 \%$ de muestras positivas a quistes y trofozoitos de Giardia.

Otros trabajos realizados sobre giardiasis en perros provenientes del cono sur de Lima Metropolitana han reportado prevalencias que fluctúan entre 8 y $16 \%$ utilizando el examen directo y la técnica de sedimentación espontánea, respectivamente, considerándose los resultados como una prevalencia moderadamente alta (Zárate, 2003); mientras que en otro estudio se encontraron grandes diferencias al evaluar la presencia de Giardia mediante la prueba de flotación con sulfato de zinc y biopsias intestinales en los mismos animales (11 y 35\%), respectivamente (Vásquez, 1989).

Los resultados obtenidos en el presente trabajo demuestran una prevalencia moderada de Giardia sp. en la Provincia Constitucional del Callao. Sin embargo, considerando que en el presente estudio sólo se evaluó una muestra por animal y no tres muestras seriadas para poder obtener una mayor sensibilidad de la prueba (Larragán, 1993), cabría esperar una prevalencia mayor por lo que el riesgo para la salud pública se encontraría incrementado. En ese sentido, se debe considerar que la población humana y canina en el Callao se ha incrementado considerablemente 
debido al fenómeno migratorio del año 2000 (Dirección de Salud-I, 2001b) y que las condiciones higiénicas y sanitarias de la población no han mejorado en los últimos años. A pesar que la variación en la prevalencia de Giardia en los distritos evaluados fue amplia (desde 3.1 a 16.7\%), no existió diferencia estadística significativa entre ellas, debido probablemente al reducido tamaño muestral por distrito.

Trabajos similares realizados en zonas urbanas de otros países y en los cuales se utilizó una sola muestra para el examen parasitológico, reportaron prevalencias moderadas, como la reportada por Jacobs et al. (2001) en Canadá (7.2\%) y Mochizuki et al. (2001) en Japón (48.2\%). Estos datos reflejan la gran variabilidad de la ocurrencia de Giardia, incluso dentro de una misma región.

En el presente estudio se halló que el $42.0 \%$ (29/69) de las muestras positivas a Giardia sp. eran diarreicas o pastosas, y mediante la prueba de regresión logística se determinó que existió una relación estadística significativa entre la presencia de Giardia y el estado diarreico o pastoso de las heces. Esto concuerda con otros trabajos como el de Ito et al. (2001) donde se detectaron Giardia sp. con más frecuencia en heces blandas y diarreicas que en heces aparentemente normales. Se debe tener en cuenta que la diarrea es un trastorno gastrointestinal que acompaña a diversas enfermedades; sin embargo, la presentación clínica de la giardiosis en perros cursa con cuadros diarreicos o heces que no son aparentemente normales, generalmente recurrentes o crónicas y en animales que adelgazan a pesar de que no pierden el apetito.

Según los análisis de regresión logística, existe una tendencia estadística de que las muestras fueran positivas dependiendo del lugar de procedencia. Se debe considerar que en la zona de estudio existen animales bien cuidados desde el punto de vista sanitario y nutricional, así como perros vagabundos que pueden servir de reservorio y fuente de infección de Giardia sp. Por otro lado, los caninos de vida intradomiciliaria o aquellos provenientes de ambientes controlados como perreras o albergues, también pueden infectarse con el parásito. Este hecho se demuestra en resultados de trabajos realizados en caninos de países desarrollados como Japón (Mochizuki et al., 2001) donde la prevalencia del parásito en animales de compañía es considerable.

\section{Conclusiones}

? Se encontró una prevalencia moderada de Giardia sp. en caninos domésticos de la Provincia Constitucional del Callao, hallando animales positivos en todos los distritos y estratos distritales en los que se divide la población de la zona. Esto constituye un riesgo para la salud de los caninos y de la población humana, especialmente la infantil.

? Es más probable encontrar quistes o trofozoitos de Giardia sp. en heces diarreicas o pastosas que en heces aparentemente normales, por lo que ante cuadros diarreicos en mascotas es conveniente consultar a un médico veterinario, para un adecuado estudio y tratamiento del animal.

\section{Literatura Citada}

1. Dirección de Salud I - Callao. 2001a. Programación Van Can 2001. Campaña antirrábica canina 2001. Oficina de Control de Zoonosis, TBC y ETS. Lima.

2. Dirección de Salud I-Callao. 2001 b. Presupuesto y Planificación 2002. p 89. Dirección de Epidemiología. Lima.

3. Furness, B.; M. Beach; J. Roberts. 2000. Giardiasis surveillance - United States, 1992-1997. Rep. CDC Surveill. Summ. 49: 1-13. 
4. Ito, N.; N. Muraoka; M. Aoki; T. Itagaki. 2001. Prevalence of Giardia lamblia in household dogs. Kansenhogaku Zasshi 75: 671-677.

5. Jacobs, S.; C. Forrester; J. Yang. 2001. A survey of the prevalence of Giardia in dogs presented to Canadian veterinary practices. Can. Vet. J. 42: 45-46.

6. Larragán, M. 1993. Comparación de los principales métodos diagnósticos para enteroparásitos. Tesis de Bachillerato. Facultad de Medicina "Alberto Hurtado", Univ. Peruana Cayetano Heredia. Lima. 50 p.

7. López, D.; E. Sagaro; M. Valdés; T. Fragoso; J. Albizu; L. Campos. 1996. Enteropathogenic agents isolate in persistent diarrhoea. Rev. Gastroenterol. Peru 16: 214-221.

8. Maco, F.V.; L. Marcos; A. Terashima; F. Samalvides Cuba; E. Gotuzzo. 2002. Distribution of entero-parasitic infections in the Peruvian highland: study carried out in six rural communities of the department of Puno, Peru. Rev. Gastroenterol. Peru 22: 304-309.

9. Meloni, B.; R. Thompson; T. Hopkins; J. Reynoldson; M. Gracey. 1993. The prevalence of Giardia and other intestinal parasites in children and aboriginal communities in the Kimberley. Med. J. Aust. 158: 157-159.

10. Mochizuki, M.; M. Hashimoto; T. Oshida. 2001. Recent epidemiological status of canine viral enteric infections and Giardia infection in Japan. J. Vet. Med. Sci. 63: 573-575.

11. Stevens, D. 1982. Giardiasis: hostpathogen biology. Rev. Infect. Dis. 4: 851-858.

12. Uribe, M.; R. Valdivia; E. Carrasco. 1997. Gastrointestinal symptoms in acquired immunodeficiency syndrome (AIDS): a review of one hundred cases at " Arzobispo Loayza” Hospital. Rev. Gastroenterol. Peru 17: 214-221.

13. Vásquez, A. 1989. Prevalencia y cultivo axénico de Giardia intestinalis en 55 perros procedentes de un área circundante a la ciudad de Lima. Tesis de Bachillerato. Facultad de Medicina "Alberto Hurtado", Univ. Peruana Cayetano Heredia. Lima. $26 \mathrm{p}$.

14. Zárate, D.; A. Chávez; E. Casas; N. Falcón. 2003. Prevalencia de Giardia sp. en canes de los distritos del Cono Sur de Lima Metropolitana. Tesis de Médico Veterinario. Rev. Inv. Vet. Perú 14: 134-139. 